\title{
Germanica
}

\section{Jaspers et son temps}

Jaspers und seine Zeit

Jean-François Robinet

\section{CpenEdition}

Journals

Édition électronique

URL : http://journals.openedition.org/germanica/2434

DOI : 10.4000/germanica.2434

ISSN : 2107-0784

Éditeur

Université de Lille

\section{Édition imprimée}

Date de publication : 31 décembre 1990

Pagination : 83-101

ISBN : 9782913857025

ISSN : 0984-2632

Référence électronique

Jean-François Robinet, « Jaspers et son temps », Germanica [En ligne], 8| 1990, mis en ligne le 27 novembre 2014, consulté le 06 octobre 2020. URL : http://journals.openedition.org/germanica/2434 DOI : https://doi.org/10.4000/germanica.2434

Ce document a été généré automatiquement le 6 octobre 2020.

(c) Tous droits réservés 


\title{
Jaspers et son temps
}

\author{
Jaspers und seine Zeit
}

Jean-François Robinet

Jaspers ${ }^{1}$ est surtout connu comme philosophe de l'existence. C'est à ce titre qu'il a agi dans la philosophie du $\mathrm{XX}^{\mathrm{e}}$ siècle. L'œuvre où se trouve l'essentiel de son système s'intitule simplement Philosophie ${ }^{2}$; elle est parue en Allemagne en 1932, quelques années après la publication de Être et Temps de Heidegger (1927). Philosophie se compose de trois volumes. Dans le premier volume, Orientation dans le monde, Jaspers montre les limites de la connaissance scientifique (dans un sens analogue à Kant) et l'insatisfaction de l'individu à l'égard de toute science de l'esprit, en quoi il critique la prétention hégélienne à opérer une totalisation « spirituelle » du monde humain. C'est à partir des limites ainsi révélées du savoir sous toutes ses formes que Jaspers découvre un champ du réel qui échappe à toute saisie objectivante : le rapport à soi dans la certitude de son unicité irréductible, ce qu'il appelle « l'existence » dans le sens singulier que l'on trouve déjà chez Kierkegaard. L'existence ainsi comprise, ne peut être connue dans un savoir apodictique, mais seulement «éclaircie » par une réflexion philosophique consciente du caractère inépuisable de l'existentialité. D'où le titre du deuxième volume: Éclairement de l'existence (existenzerhellung), où Jaspers fait l'analyse des principales catégories existentielles : la liberté, la communication, l'historicité, la situation. Dans le troisième volume intitulé Métaphysique, Jaspers récuse la prétention objectivante de la métaphysique classique pour en retenir le mouvement de transcendance. L'existence est tension du fini vers l'infini. Les discours humains sur l'absolu: mythes, religions, métaphysiques spéculatives, peuvent donc être interprétés comme des symboles du mouvement existentiel de transcendance, ce que Jaspers appelle des « chiffres ».

2 Par cet ouvrage Philosophie, Jaspers apparaît comme un des fondateurs avec Heidegger de l'existentialisme contemporain. On sait que Heidegger a refusé l'étiquette « existentialiste » et dans la suite de son œuvre il a bien montré que l'analytique de l'existence développée dans Être et Temps était une étape préparatoire pour une interrogation ontologique du sens de l'être. D'où le travail de Heidegger : déconstruire la métaphysique classique oublieuse de la finitude et se mettre à l'écoute des poètes, puisque la poésie est le langage immédiat où se révèlent les voix de l'être. L'orientation 
suivie par Jaspers est significativement différente. Jaspers essaye d'élaborer une systématique du réel à travers les catégories méthodologiques de la connaissance. Tel est le projet exposé dans De la Vérité, paru en 1948, qui porte le sous-titre ambitieux de Logique Philosophique, premier volume. Les trois autres volumes annoncés ne paraitront jamais! Cette orientation montre que Jaspers s'intéresse aux médiations du réel, au lieu d'exténuer le discours dans le silence attentif des voix raréfiées de l'être, à la manière de Heidegger. La philosophie de Heidegger reste une philosophie de l'individu ${ }^{3}$, et dans cette mesure on ne trouve pas en elle de philosophie politique développée et positive ; par contre, la philosophie de Jaspers contient une philosophie politique développée et une philosophie de l'histoire. C'est cette philosophie qui nous intéresse et que nous allons essayer d'interpréter.

3 Pour ce faire, il nous suffit de suivre l'itinéraire de Jaspers à travers la publication de ses œuvres politiques. La première étape est constituée par la publication en $1931 \mathrm{du}$ petit livre: La situation spirituelle de notre époque ${ }^{4}$. Ce livre est contemporain de la philosophie de l'éclairement de l'existence. Jaspers regarde le champ politique de l'extérieur à partir d'un point de vue moraliste et aristocratique qui ressemble fortement à la perspective nietzschéenne. C'est pourquoi nous proposons de comprendre cette étape sous le titre : «Jaspers, médecin de la civilisation ».

4 La deuxième étape de la pensée jaspersienne du politique est constituée par les gros traités: Origine et sens de l'histoire ${ }^{5}$, publié en 1949, La Bombe atomique et l'avenir de l'humanité $e^{6}$ publié en 1958. Dans ces livres Jaspers développe une pensée politique concrète, soucieuse à la fois du devenir de l'universalité dans l'histoire et de l'analyse positive des problèmes de la politique mondiale contemporaine. Ces livres coïncident avec un attrait de plus en plus marqué de Jaspers pour la philosophie kantienne de la raison et son projet cosmopolitique. Nous proposons de désigner cette étape par le titre : « Jaspers, citoyen du monde ».

5 À l'intérieur de cette deuxième étape, nous ferons une place à part à un ensemble d'opuscules que Jaspers publie à partir de 1945 jusqu'à sa mort, par lesquels il prend directement parti dans la politique allemande de son temps : La culpabilité allemande $(1946)^{7}$, Liberté et réunification $(1960)^{8}$, Vers où dérive la République Fédérale? (1966) ${ }^{9}$. Nous proposons d'analyser ces écrits sous le titre : « Comment être allemand après Hitler? ».

\section{Jaspers, médecin de la civilisation}

6 Jaspers, de son propre aveu, ne s'est d'abord pas intéressé à la politique. "Jusqu'en 1914, je restais résolument apolitique. Tout semblait alors stable. [...] Je m'adonnais uniquement aux tâches intellectuelles $»^{10}$. Avec la guerre qui commence en 1914 Jaspers comprend que l'histoire politique touche tous les hommes, malgré eux. C'est dans les conversations avec Max Weber qu'il fréquente régulièrement de 1905 jusqu'à sa mort en 1920, qu'il acquiert une conscience politique.

7 En 1929 il accepte la proposition des éditions Göschen d'écrire le millière fascicule de la collection sur le thème des mouvements spirituels de l'époque. Il achève le livre en 1930, avant les élections de septembre, où le parti national-socialiste remporte son premier succès. La Situation spirituelle de notre époque est publié un an plus tard, en octobre 1931, quelques mois avant la publication du maître ouvrage Philosophie. La Situation spirituelle est le développement pour le grand public d'une partie du système, 
celle où Jaspers analyse ce qu'il appelle « les figures de l'objectivité ", dans la quatrième division du volume « l'éclairement de l'existence ».

Conformément à l'esprit de sa méthode, Jaspers commence par récuser deux manières d'appréhender une situation historique : l'illusion d'une connaissance synthétique du tout qui oublie la finitude de la connaissance humaine, l'absolutisation dogmatique d'une perspective finie qui oublie l'orientation vers la totalité. La compréhension philosophique est à la fois personnelle, dans la mesure où elle engage l'individu, perspectiviste, pour tenir compte de la richesse du réel et de ses antinomies irréductibles, totalisante, car si la totalité achevée échappe à l'esprit humain, toute vérité doit se situer dans l'horizon d'une totalité recherchée.

Jaspers fait d'abord l'analyse de la société moderne. Cette société est caractérisée par la technique qui permet d'exploiter la nature, l'organisation qui permet de rationaliser les tâches économiques et le formalisme juridique qui permet d'introduire la prévision dans les rapports sociaux, enfin par le phénomène des masses. Jaspers distingue trois sens du mot "masse». Dans un premier sens, la masse peut désigner une foule inorganisée, avec les caractères que la sociologie de Le Bon a mis en évidence: la suggestibilité, l'intolérance, la mobilité. Dans un deuxième sens, la masse désigne le public, c'est-à-dire une opinion anonyme qui ne s'incarne dans aucun individu déterminé. Dans un troisième sens, la masse désigne un ensemble d'hommes dans une organisation, par exemple le prolétariat ou le corps médical, dans la mesure où la majorité numérique détermine le comportement des individus. "Les groupes qui décident de ce qui arrive, soit par leur activité silencieuse, soit par référendum organisé, constituent une masse lorsque l'individu n'y joue que le rôle d'un numéro ${ }^{11}$. Ces distinctions étant faites, sans que Jaspers s'y tienne d'ailleurs avec rigueur, l'essentiel des analyses porte sur la tension entre la masse et l'individu. La société tend à réduire l'individu à une fonction et perçoit les valeurs de l'individualité comme une sorte de résidu irrationnel que le progrès doit éliminer. Elle admet ces valeurs dans des domaines limités: vie amoureuse, aventure, jeu, sport et les combat dès que l'individualité s'abandonne à l'angoisse vitale et au dégoût à l'égard du travail. La société moderne demande à l'individu d'être un travailleur performant à l'intérieur du système qui est organisation d'organisations. Celui qui se soumet à sa logique réussit, certes, mais les dirigeants ne sont que les fonctionnaires des masses !

La société se heurte à une double limite. Elle aspire à devenir un mécanisme rationnel parfait de travail et de prévoyance, mais elle est continuellement traversée par des irrationalités nouvelles et diverses : problèmes démographiques, sociaux, économiques, techniques, problèmes d'environnement, etc. Même si, par impossible, la rationalisation aboutissait, l'individu resterait insatisfait, car il ne peut se réduire à être uniquement un travailleur et un consommateur, sauf en de rares instants.

11 À partir de cette double limite se manifeste la nécessité de l'État. L'État est une organisation dans laquelle la société divisée par des intérêts divers, peut arriver à une compréhension unifiée d'elle-même. L'État est aussi organisation de la communauté, ce qui donne à l'individu un enracinement concret dans la tradition d'une histoire et dans une appartenance populaire. L'État n'est pas seulement organisation, appareil de contrainte qui détient le monopole de la violence légitime, selon la définition de Max Weber, il porte en lui-même un sens positif dans la mesure où il est l'acteur du destin historique de la nation. 

premier est celui des rapports internationaux. La guerre est toujours possible pour régler les rapports entre États. Mais la guerre moderne n'apporte plus rien de décisif, « elle n'est plus manifestation d'une noblesse humaine qui combat pour son avenir ${ }^{12}$. Cependant Jaspers reconnaît qu'une paix définitive est utopique. D'où cette prophétie : « Nous restons menacés d'une nouvelle guerre, plus terrible que toutes les autres, qui signifierait la fin de l'Europe actuelle $»^{13}$. Le deuxième problème est le problème de la démocratisation intérieure. Comment faire accéder les masses à une responsabilité politique qui soit à la fois participation active et lucidité ? D'où le problème de l'éducation, éducation scolaire d'abord qui ne doit pas être seulement transmission de connaissances utilitaires, mais aussi communication de la culture historique, éducation politique ensuite qui doit être le fait de l'État. Jaspers dessine alors le portrait de ce qu'il appelle «le vrai chef, capable de montrer la voie et d'entreprendre une action continue ", et il l'oppose au chef de coalition parlementaire, réduit à gérer des politiques de compromis à court terme, et au démagogue qui exploite les bas instincts des masses populaires.

Mais l'État n'est pas le fondement dernier de la vie humaine, l'État sous peine de se réduire à une pure organisation juridique et à une technique du pouvoir, renvoie à des enjeux culturels, à ce que Jaspers appelle le « Geist », terme qui n'a guère en français de traduction dotée de la même richesse connotative. Jaspers consacre la troisième et la quatrième partie du livre à l'analyse des mouvements «spirituels» de son temps. Le tableau que fait Jaspers est très sombre : la culture est menacée par le nivellement et la facilité, par le "journalisme»; l'art est tenté de se réduire à un divertissement populaire; les grandes doctrines où les hommes tentent de trouver la vérité de leur monde : le marxisme, le freudisme, l'anthropologie, sont menacées par la perversion idéologique et le dogmatisme. De nouvelles croyances laïques se substituent à l'ancienne foi religieuse. L'homme moderne est un homme privé de l'appui d'une foi révélée, et les sciences ne peuvent répondre à son désir de sens.

Dans la cinquième partie du livre Jaspers propose quelques orientations positives. $\mathrm{Du}$ point de vue politique il est très difficile de donner des repères sûrs, étant donné la complexité du monde et les limites de notre compréhension. Le seul repère sûr est «l'opposition qui sépare l'esprit occidental de l'esprit asiatique, la liberté européenne $\mathrm{du}$ fanatisme russe $»^{14}$. Du point de vue moral il s'agit de constituer une nouvelle noblesse qui ne soit ni de naissance, ni d'argent, mais de cœur et d'esprit. Quant à la philosophie, elle doit sortir de son académisme et devenir philosophie de la vie. La philosophie de l'existence offre à l'individu la possibilité d'accéder à la subjectivité véritable en rapport avec la transcendance.

arquons d'abord que Jaspers fait de larges emprunts à la sociologie de Max Weber la rationalisation, le désenchantement du monde moderne, la bureaucratie, le rôle du chef, la définition de l'État. Cependant Jaspers ne suit pas son maître jusqu'au bout, il ne sépare pas totalement l'objectivité de la liberté, il ne pense pas que le choix des valeurs relève d'une décision injustifiable et irrationnelle ${ }^{15}$.

Remarquons également que Jaspers est singulièrement aveugle à l'égard de certains phénomènes de l'époque : il ne parle guère des problèmes sociaux et de la lutte des classes, il paraît peu connaître la création littéraire et artistique du début du $\mathrm{XX}^{\mathrm{e}}$ siècle. Il parle de Piscator et du «Bauhaus», il ne parle pas de Kafka, de Proust, de Thomas 
Mann. Il passe également sous silence la formidable aventure picturale qui se joue dans les premières décennies du siècle, le passage de la peinture figurative à la peinture abstraite avec des peintres comme Picasso ou Kandisky.

Mais on ne juge pas uniquement un livre en fonction de ses emprunts ou de ses lacunes, il s'agit d'en apprécier la thèse centrale. Risquons ce jugement global: Situation spirituelle est un livre de moraliste. Jaspers fait la critique de son temps en faisant le recensement de tous les traits qui constituent le monde moderne dans sa spécificité : technique, économie, massification. La critique de Jaspers s'inscrit en ce sens dans toute une tradition que l'on peut faire remonter à Rousseau, que Jaspers cite d'ailleurs. Déjà Jean-Jacques Rousseau, au XVIII ${ }^{e}$ siècle, avertit ses contemporains de ne pas s'abandonner à l'illusion du progrès matériel et lance un appel à la conversion morale. La mise en cause systématique de l'Aufklärung est le fait du romantisme allemand et de tout un courant que l'on appelle la « Kulturkritik » qui a fleuri tout particulièrement en Allemagne dans la seconde moitié $\mathrm{du} \mathrm{XIX}^{\mathrm{e}}$ et la première moitié $\mathrm{du} \mathrm{XX}^{\mathrm{e}}$ siècle. Citons, parmi les ouvrages les plus connus, Contribution à une critique de notre temps de Walter Rathenau (1912), Le Déclin de l'Occident d'Oswald Spengler (1918-1922), Malaise dans la civilisation de Freud (1931). Jaspers s'inscrit incontestablement dans ce courant, mais il prend distance à l'égard du groupe des « conservateurs révolutionnaires » qui prône le retour à un passé communautaire mythique pour lutter contre le capitalisme et le communisme. Il ne fait pas appel à la vie instinctive individuelle ou collective, aux liens du sang ou à un quelconque mysticisme, mais à la dignité de l'individu, à sa noblesse, et à une communication des individus dégagés des contraintes des appareils idéologiques ${ }^{16}$. L'opposition centrale du livre entre l'existence possible (Existenz) et la réalité empirique (Dasein) contient virtuellement l'opposition entre l'entendement (Verstand) et la raison (Vernunft) qui va devenir le thème privilégié de la philosophie ultérieure de Jaspers.

\section{Jaspers, citoyen du monde}

La pensée politique de Jaspers s'infléchit très nettement après 1945. L'expérience du nazisme, la menace que fait peser le régime sur sa vie (en 1937 le gouvernement allemand interdit officiellement à Jaspers d'enseigner du fait de son mariage avec une Juive), la difficulté d'être allemand, tout cela conduit Jaspers à une modification de son rapport au politique. D'une manière générale Jaspers prend distance à l'égard de sa philosophie de l'existence et de ce que cette philosophie peut comporter de " hauteur » moralisante, de pathos nietzschéen, pour s'engager dans une philosophie concrète de l'histoire et une philosophie politique soucieuse des valeurs d'universalité. La référence à Kant devient de plus en plus centrale dans les œuvres philosophiques d'après guerre.

Dans Origine et sens de l'histoire, publié en 1949, Jaspers fait un tableau grandiose de l'histoire universelle. Il prolonge ainsi les philosophies de l'histoire de Kant et de Hegel, mais il tient également compte des travaux de Spengler, de Toynbee et d'Alfred Weber.

L'originalité de Jaspers consiste à se décentrer d'une conception de l'histoire qui prend le christianisme comme axe de l'histoire. Le christianisme est une religion propre aux peuples de l'Occident, une religion locale donc, que l'on ne peut prendre comme repère de l'histoire universelle sans risquer d'être accusé d'ethnocentrisme. Jaspers propose un axe de l'histoire qui est étonnamment commun aux trois grands lieux de civilisation que sont l'Occident, l'Inde et la Chine. Vers le troisième millénaire avant Jésus Christ 
sont apparues des civilisations historiques et socialement différenciées autour des grands fleuves chauds: le Nil, la Mésopotamie, l'Indus, l'Hoang-Ho. Mais ces civilisations se sont figées dans des structures, à la fois politiques et religieuses, peu favorables à l'évolution. C'est dans une période historique située entre 800 et 200 avant notre ère que sont découverts en trois lieux du monde, de manière probablement totalement indépendante, ce que Jaspers appelle «les fondements spirituels de l'humanité ». En Chine vivent Confucius et Lao-tseu qui développent réflexivement une morale populaire et aristocratique. Aux Indes apparaissent deux grandes religions : le bouddhisme et l'hindouisme brahmanique dans les Upanishads. En Perse, Zarathoustra développe sa religion de la pureté et de la lumière. En Palestine se lèvent les grands prophètes : Elie, Esaïe, Jérémie. En Grèce enfin c'est l'époque où naît un genre culturel que les Grecs vont appeler « philosophie » où s'illustrent Parménide, Héraclite, Socrate, Platon, mais c'est aussi l'époque des grands écrivains (les tragiques) et des grands savants (Thucydide, Archimède). D'une manière générale cette époque de l'histoire est caractérisée par le fait que les hommes mettent en question les mythes et les coutumes jusqu'alors implicitement reconnus et incontestés et élaborent réflexivement de nouvelles doctrines et de nouvelles religions où l'homme semble pour la première fois s'éveiller à lui-même et au monde. L'inquiétude à l'égard de l'être, de la vérité, de la justice ou du salut ouvre à la pensée humaine un champ infini de possibilités.

C'est durant la période axiale qu'eut lieu la révélation de ce qu'on nomma plus tard raison et personnalité. Les résultats obtenus par l'individu ne se transmettent nullement à tout le monde. À cette époque, la distance qui séparait de la multitude ces hautes figures était immense. Mais le progrès individuel entraîne indirectement celui de tous. La condition humaine progresse d'un bond ${ }^{17}$.

Depuis la période axiale, Jaspers considère que la seule grande rupture dans l'histoire est l'invention en Europe à la fin du moyen âge de la science et de la technique modernes. Cette invention va établir l'hégémonie de l'Europe sur le monde et mettre en contact les civilisations qui vivaient dans l'ignorance les unes des autres. Les temps modernes nous font entrer dans une histoire qui est désormais "mondiale » et qui se substitue aux histoires locales antérieures.

La philosophie de l'histoire, par la mise en perspective des "percées" historiques décisives, nous permet de prendre une juste conscience de notre situation, situation créée par la civilisation scientifico-technique qui devient planétaire. Jaspers repère dans notre monde trois problèmes fondamentaux que l'homme moderne doit résoudre sous peine de sombrer dans la confusion et la violence : le problème social et la liberté politique, la constitution d'un ordre mondial pacifique, l'exigence d'une foi nouvelle. Résumons rapidement les analyses de Jaspers sur ces trois questions en notant au passage les infléchissements par rapport aux analyses des années 1930.

24 Jaspers ne regarde plus le monde économique moderne comme étant une menace permanente de perdition pour l'individualité, mais comme une condition effective de liberté.

Tous les hommes doivent disposer des biens nécessaires à la vie, et prétendent jouir d'une existence normale. [...] Le socialisme est la tendance universelle de l'humanité présente, réclamant l'organisation du travail et une part aux résultats, afin de réaliser la liberté pour tous. À ce degré, presque tout le monde est socialiste $^{18}$.

Mais le socialisme doit intégrer les principes de la liberté politique et doit éviter la tentation $d u$ "communisme scientifique». Le projet d'une économie dirigée et 
totalement planifiée repose, selon Jaspers, sur deux illusions, la croyance selon laquelle on peut organiser la vie humaine selon le modèle de la technique rationnelle et l'illusion d'une connaissance totale de l'histoire. Il faut reconnaittre les limites de notre pouvoir et de notre connaissance. Dans cette distinction entre deux manières de concevoir l'histoire, on peut reconnaître, au delà d'une défense du principe d'une économie de marché, la différence qui va devenir centrale dans la pensée de Hannah Arendt entre "agir » et " faire ", telle qu'elle est exposée dans La Condition de l'homme moderne. Hannah Arendt montre que l'« action » politique (pensée dans sa vérité par les Grecs) se corrompt dès lors qu'elle est pensée à travers le modèle de la fabrication technique. Dans le modèle de la fabrication, les hommes sont pris comme matériau par une force extérieure. En ce sens il est possible de dire que la tentation totalitaire est inhérente à la pensée technique ${ }^{19}$.

Sur le thème de la liberté politique, Jaspers reprend les idées contenues dans Situation spirituelle, mais il comprend désormais la liberté politique dans l'opposition avec le totalitarisme qu'il définit comme domination totale ( totale Herrschaft»), fondée sur la confusion de l'État et de la société, une politique perpétuelle du mensonge et de la force et des hommes qui ne connaissent pas une tradition de liberté. Le totalitarisme est le maître d'hommes sans maîtrise intérieure, d'hommes qui ne connaissent pas la liberté personnelle, et qui sont donc les victimes privilégiées de la propagande totalitaire. Une fois encore, on peut reconnaître dans la pensée politique de Jaspers de nombreuses ressemblances avec la pensée d'Hannah Arendt. Jaspers fait d'ailleurs souvent allusion au livre d'Hannah Arendt, Le Système totalitaire (1951), et lui reconnaît le mérite de l'avoir le mieux éclairé sur la question.

Le deuxième problème que Jaspers développe est celui de la paix mondiale. Ce problème lui tient tellement à cœur qu'il lui consacre tout un livre de près de cinq cents pages: La Bombe atomique et l'avenir de l'humanité. L'arme atomique depuis son apparition sur la scène mondiale met plus que jamais les hommes en urgence de réfléchir sur les possibilités d'éviter la guerre et d'instaurer un ordre juridique mondial, conforme à l'idée que Kant exprimait déjà de manière parfaitement claire à la fin du XVIII ${ }^{e}$ siècle ${ }^{20}$. L'idée d'une paix juridique n'est pas une pure utopie. Il faut reconnaître les limites de la politique «Pure » qui ne vise que l'intérêt et la puissance. La politique qui fait progresser l'histoire (ce que Jaspers appelle la " grande politique ») se comprend comme réalisation dans l'histoire d'un principe supra-politique: l'exigence morale et la raison. Jaspers analyse alors très concrètement les situations où l'on perçoit bien que l'idéalisme moral (les droits de l'homme, l'appel à la raison) agit effectivement sur le réalisme politique. La paix mondiale demande deux conditions. Il faut d'abord que l'Occident renonce loyalement et sans arrière-pensée au colonialisme et établisse des relations de coopération librement consenties avec les pays sousdéveloppés. Il faut ensuite établir une confédération mondiale des États. L'O.N.U. depuis sa création est l'objet de multiples critiques. On peut lui reprocher d'être inefficace, de représenter les intérêts des grandes puissances, d'être une tribune de propagande. Cependant «l'O.N.U. est plus que rien $»^{21}$. Aussi mauvais qu'en soit le fonctionnement, c'est un moyen d'action en faveur de la paix. La constitution d'un véritable ordre juridique mondial demande une conversion ("Umkehr») radicale des hommes et des politiques traditionnelles. Ainsi se dessine la communauté des hommes raisonnables (Gemeinschaft der Vernünftigen) qui, à travers les frontières et les 
institutions rivales, établissent une communication libre de tout rapport de force et de tout mensonge.

Le troisième problème que Jaspers développe est le problème de l'avenir de la foi. Il faut bien reconnaître le déclin des églises dans le monde moderne. Les idéologies politiques ont prétendu remplacer la foi religieuse par des objectifs terrestres. Mais les idéologies du progrès ne tiennent pas compte, selon Jaspers, de la profondeur spirituelle de l'homme. C'est pourquoi il faut restaurer l'idée authentique de la transcendance. La foi est projection de l'homme vers l'éternité et vers les possibles dans le monde qui prennent source dans la liberté.

La foi est l'englobant qui nous dirige, même quand l'entendement semble se suffire à lui-même. Elle ne consiste pas en un credo défini, en des dogmes - le dogme ne peut être que son expression historique ; il peut aussi induire en erreur. La foi est ce qui nous comble et nous anime, ce qui est au fond de l'homme, ce par quoi l'homme, s'élevant au-dessus de lui-même, se rattache à l'origine de l'être ${ }^{22}$.

Jaspers pense que la foi future peut encore se nourrir des valeurs de la période axiale, d'où proviennent les religions issues de la Bible. L'ennemi perpétuel de la foi, quelle que soit sa forme, est le nihilisme, qui déclare que toute activité est vaine et que toute vérité est illusoire. L'alternative entre foi et nihilisme est l'alternative la plus radicale et la plus profonde qui traverse toute l'histoire.

On voit maintenant l'originalité de la philosophie jasper sienne de l'histoire, à la fois par rapport aux philosophies kantiennes et hégéliennes, et par rapport à ses propres conceptions des années 1930. Jaspers insiste sur le fait que l'histoire est l'aventure de la liberté agissant par des percées décisives qui peuvent toujours être perdues dans la mesure où les hommes ne sont plus capables d'assumer l'héritage de leur origine spirituelle. Il montre que l'histoire actuelle est traversée par des antinomies qui constituent les lignes de force de notre monde, dans lequel les hommes doivent faire des choix décisifs : socialisme ou planisme, liberté politique ou domination totale, paix perpétuelle ou destruction de l'humanité dans la guerre atomique, foi ou nihilisme. Le philosophe a pour rôle de formuler clairement les enjeux fondamentaux de notre situation et de les présenter aux hommes qui sont par leur activité immergés dans la particularité et oublient de porter leur regard sur l'universel. Ainsi, en faisant prendre conscience des vrais problèmes aux hommes de son temps, le philosophe est-il acteur de l'histoire, d'une histoire qui englobe maintenant tous les hommes, il est véritablement citoyen du monde.

\section{Comment être Allemand après Hitler?}

31 Nous avons vu que Jaspers a évolué depuis 1930. Il est passé d'une philosophie « aristocratique » de la subjectivité à une philosophie soucieuse d'articuler la morale et la politique de manière positive dans une "politique morale", tout en gardant l'autonomie des deux sphères. Dans le langage hégélien, on pourrait dire que Jaspers est passé de la morale (Moralität) à l'éthique (Sittlichkeit). Dans cette perspective une question va devenir de plus en plus préoccupante, c'est la question du destin de l'Allemagne et de la germanité. Jaspers intervient plusieurs fois après 1945, par des prises de positions publiques, sur la «question allemande». Ses prises de position ont suscité des réactions vives et, parfois, des incompréhensions dans l'opinion publique. 

le semestre d'hiver 1945-1946 sur la «situation spirituelle en Allemagne». Les Allemands doivent faire leur examen de conscience afin de clarifier le sentiment obscur de leur faute et ainsi se libérer du passé pour prendre un nouveau départ. distingue quatre types de faute. $\mathrm{La}$ « faute criminelle » réside dans l'infraction à l'égard de la loi et relève des tribunaux. Il est normal que les responsables nazis soient punis et cette justice appartient aux vainqueurs qui en ont acquis le droit. Jaspers légitime ainsi les procès de Nuremberg. La "faute politique » est le fait de l'État, mais l'État repose sur chaque citoyen qui lui donne son soutien. Celui qui n'a pas émigré, qui ne s'est pas exposé pour résister aux crimes, est politiquement responsable. Assumer cette responsabilité c'est se soumettre à la justice du vainqueur, car celui qui a fait recours à la force doit accepter d'en subir la loi! Il faut donc accepter la tutelle étrangère et la partition de l'État allemand. La "faute morale » est l'âme de la faute criminelle et politique. Le mal n'est possible qu'à partir d'une perversion de la conscience individuelle, d'un refus de se subordonner à l'universel, en l'espèce d'avoir fait passer le fait d'être Allemand avant le fait d'être homme. Il revient à chaque Allemand de ne pas avoir voulu distinguer entre le patriotisme et le nationalisme aveugle, le service de l'État et l'obéissance inconditionnelle. La "faute métaphysique » est la plus générale et la moins circonstanciée. Elle tient à la condition humaine ellemême, elle s'apparente à ce que les chrétiens appellent «le péché originel » et ce que Kant appelle « le mal radical ». Dans la condition humaine il faut bien reconnaître une tendance irréductible vers le mal qui fait que les hommes sont tentés par la force, les passions obscures et le mensonge. quelques uns, mais surtout de la faute politique et morale qui touche presque tous les Allemands. Jaspers estime à 100000 le nombre des Allemands qui ont fait de la résistance intérieure au nazisme et qui ne sont donc pas compromis (unbelastet). C'est parmi eux que devraient être recrutés les cadres de la République Fédérale naissante.

Liberté et réunification est un livre composé d'une interview avec Thilo Koch et d'articles publiés dans la revue Die Zeit au cours de l'année 1960. Jaspers soutient la thèse selon laquelle les Allemands doivent se soucier davantage de réaliser la liberté politique dans la République Fédérale que de penser à la réunification des deux Allemagnes et aux frontières de l'Etat bismarckien. Bismarck a construit l'État "prusso-allemand » par «le sang et le fer ». Et Hitler voulait englober l'ensemble des pays germanisés. Mais à chaque fois il s'agit de politique fondée sur la force. Pendant ces périodes de l'histoire les Allemands n'ont guère appris la démocratie. L'État de Bismarck établit une démocratie factice: le monarque se permet un comportement arbitraire; les fonctionnaires sont compétents, mais subordonnés au gouvernement; les partis sont incapables de fournir de véritables hommes d'État. La république de Weimar n'a guère le temps d'instaurer des mœurs politiques démocratiques; étant née de la défaite, elle fut d'emblée discréditée aux yeux de la majorité des Allemands. L'armée et les grands partis politiques (nationaux et communistes), majoritaires à partir des années 1930, ont tout fait pour la détruire. Quant à l'Allemagne Fédérale, elle a certes connu un formidable essor économique, mais l'esprit de la constitution n'a pas encore pris racine dans le peuple. «Il faut, dit Jaspers, que la loi fondamentale devienne une constitution vivante ». Et pour ce faire il faut abandonner l'idée nationaliste que le thème de la réunification continue à entretenir. Il faut accepter les frontières de 1945, l'abandon de 
la Prusse orientale et d'une partie de la Silésie, aussi dur cela soit-il. Il faut abandonner les prétentions nationalistes et se convertir à une idée "apolitique» de la grande Allemagne. La grande Allemagne comprend tous les hommes qui parlent l'allemand ou un de ses dialectes. La Hollande, la Suisse et l'Autriche en font partie.

Cette grande Allemagne, une et unique, est un concept non politique. C'est pourquoi la conscience qu'on peut en avoir précède ou dépasse la politique. Au cours des siècles, elle donna naissance sur ce plan à plus d'une formation politique, mais aucune d'entre elles ne peut prétendre représenter cette Allemagne unique ${ }^{23}$.

Au-dessus des États Allemands particuliers, il y a l'espace supérieur de l'esprit allemand qui comprend Erasme, Spinoza, Rembrandt et Burkhardt. Au-delà de la "Staatsnation " se trouve la «Kulturnation». L'Allemagne est " un peuple de peuples », se plait à répéter Jaspers après Schelling.

On comprend que Jaspers ait heurté de nombreux Allemands. Cependant il a induit un débat dans les années soixante en Allemagne qui a permis par la suite la mise en œuvre de l'« Ostpolitik» par Willy Brandt.

Vers où dérive la République Fédérale (1966) est le dernier ouvrage conséquent de Jaspers, où il prend publiquement part au débat politique de son temps. Il reproche à la démocratie allemande d'être confisquée par le pouvoir oligarchique des partis politiques («Oligarchie einer Parteienherschaft»). Les partis visent leur propre conservation, ils organisent des élections sans débat véritable et maintiennent ainsi le peuple dans la minorité, ils ne laissent aucune chance à de nouveaux partis de se constituer. Ainsi le régime démocratique cache en fait une structure oligarchique, qui contient virtuellement le risque d'un acheminement vers la dictature.

Dans cette analyse de la République Fédérale, on reconnaît un thème privilégié de la pensée politique de Jaspers: l'exigence d'une liberté politique réelle qui s'inscrirait dans le cœur des citoyens et l'exigence d'une éducation politique. Du point de vue historique, il est vrai que les mœurs des grands partis politiques et les gouvernements successifs de coalition sont peut être responsables de l'émergence dans les années 68 d'un grand mouvement de contestation qui va se constituer à la fois contre et en dehors des structures politiques traditionnelles. Cependant certains observateurs ont reproché à Jaspers de se donner la partie facile en refusant d'admettre que les partis politiques, quels que soient par ailleurs leurs défauts, occupent une place nécessaire dans la vie politique d'une nation en servant de relais entre le gouvernement et les citoyens ${ }^{24}$.

Nous avons suivi volontairement Jaspers au fil de ses publications majeures de philosophie et d'analyse politiques. Incontestablement Jaspers a modifié son approche de la réalité politique. Il fait d'abord la critique radicale de la société moderne à partir de la valeur absolutisée de la subjectivité conçue comme rapport infini et autosuffisant à soi-même (Existenz). Puis, après 1945, il élabore une philosophie positive de l'histoire où la rationalité de l'entendement est intégrée dans le devenir concret de l'universel. Initialement connu dans les seuls milieux philosophiques et universitaires, Jaspers devient dans l'Allemagne Fédérale une figure publique dont les jugements font autorité. Il s'adresse d'ailleurs régulièrement au grand public par des petits textes de vulgarisation, des émissions radiophoniques et des articles de journaux. Jaspers pense, en effet que la philosophie est liée substantiellement à la politique.

Une philosophie montre ce qu'elle est en se manifestant dans sa pensée politique. Loin d'être accessoire (beiläufig) ce fait est primordial. Ce n'est pas un hasard si le national-socialisme et le bolchévisme voient dans la philosophie leur ennemi mortel ${ }^{25}$. 
41 Heidegger et Jaspers étaient d'abord proches l'un de l'autre, par leur refus de la philosophie " universitaire ", et par leur découverte commune de Kierkegaard. Leur différence philosophique est devenue rupture insurmontable, à partir de 1933, malgré les tentatives de "médiation " d'Hannah Arendt ${ }^{26}$. La différence totale d'appréciation du nazisme peut s'expliquer par deux raisons. Jaspers, à la différence de Heidegger, ne voit pas dans la science et la technique une rationalité agressive et vide de sens, mais de la raison, bien que de la raison incomplète. Pour Jaspers la communication est certes une entreprise difficile et souvent décevante, mais en même temps c'est le lieu nécessaire de compréhension et de collaboration des hommes dans la recherche de la vérité ; alors que pour Heidegger la communication humaine est radicalement affectée par l'inauthenticité, sauf à être sauvée par la révélation poétique de l'être. Où l'on voit que Jaspers avait déjà dans sa philosophie de l'éclairement de l'existence les éléments qui lui ont permis par la suite de constituer une philosophie politique positive, qui tourne autour des valeurs humanistes de démocratie, de droits de l'homme et de paix mondiale ${ }^{27}$

Jaspers est bien philosophe de la liberté : de son mystère qui ne peut être jamais éclairci et nous préserve ainsi des dogmatismes qui prétendent enfermer l'histoire dans une loi à priori; de ses risques tragiques, car les hommes peuvent toujours préférer la « passion de la nuit » à la « loi du jour »; de sa grandeur puisque la liberté est pour l'individu et la communauté l'espace véritablement humain d'habitation du monde. Jaspers est sûrement au $\mathrm{XX}^{\mathrm{e}}$ siècle le philosophe qui se rapproche le plus de Kant.

\section{NOTES}

1. - Karl Jaspers est né en 1883 à Oldenburg, dans le nord de l'Allemagne, près de Brème, non loin des côtes de la mer du nord. Il a fait de brèves études de droit, puis des études de médecine, surtout à Heidelberg, où il obtient son doctorat en 1909. Il est d'abord assistant à la clinique psychiatrique de Heidelberg, puis après avoir soutenu sa thèse Psychopathologie générale en 1913, il devient maître de conférences en psychologie à la Faculté des Lettres, enfin en 1921 il obtient une chaire de philosophie, toujours à Heidelberg. Pendant toute l'époque nazie il doit supporter de nombreuses interdictions : interdiction de participer à l'administration de l'Université dès 1933, d'enseigner à partir de 1937, de publier en 1938. Les autorités lui reprochent de ne pas faire acte d'obédience au régime et d'être marié avec une Juive. Jaspers vit donc pendant douze années dans la peur de la déportation et de la mort. En 1945 il retrouve sa chaire de philosophie avec l'agrément des autorités américaines. En 1948 il accepte l'invitation de l'université de Bâle de prendre le poste de philosophie, celui qu'avait occupé Nietzche, où il enseigne bien au-delà de sa retraite en 1961. En 1968 il obtient la nationalité suisse. Il meurt à Bâle en 1969, à l'âge de quatrevingt-six ans. Si l'on veut comprendre les événements importants de sa vie : la maladie avec laquelle il a toujours dû lutter, sa relation privilégiée avec sa femme: Gertrud Mayer, ses rapports avec ses collègues universitaires, ses amitiés, on peut lire le petit texte Autobiographie philosophique (Stuttgart, 1956, trad. P. Boudot, Paris, 1963).

2. - Philosophie, Berlin, 1932. Trad. J. Hersch, 1986. 
3. - Voir sur cette question les articles de Karl Löwith et d'Eric Weil dans les Temps modernes, parus en novembre 1946 et en juillet 1947. Voir également dans cette revue l'article de Jean Quillien, « Le cas Heidegger. Philosophie et politique ».

4. - Die geistige Situation der Zeit, Berlin, 1931. Trad. W. Biemel et J. Ladrière, Paris, 1950.

5. - Von Ursprung und Ziel der Geschichte, Munich, 1949. Trad. H. Naef, Paris, 1954.

6. - Die Atombombe und die Zukunft des Menschen, Munich, 1958. Trad. E. Saget, Paris, 1963.

7. - Die Schuldfrage, Heidelberg, 1946. Trad. J. Hersch, 1948.

8. - Freiheit und Wiedervereinigung, Munich, 1960. Trad. J. Hersch et H. Naef, Paris, 1962.

9. - Wohin treibt die Bundesrepublik, Munich, 1966. Antwort zur Kritik meiner Schrift: Wohin treibt die Bundesrepublik, Munich, 1967.

10. - Autobiographie philosophique, p. 117.

11. - Situation spirituelle, p. 46.

12. - Ibidem, p. 111.

13. - Ibidem, p. 112.

14. - Ibidem, p. 130.

15. - Cf. François Léger, «Karl Jaspers et Max Weber» in Situation de l'homme et histoire de la philosophie dans l'œuvre de Karl Jaspers, Nancy, 1986.

16. - Signalons qu'une des premières recensions en français de Situation spirituelle se trouve dans Revue philosophique de 1933 sous la signature de Kojevnikoff, plus connu par la suite sous le nom d'Alexandre Kojève.

17. - Origine et sens de l'histoire, p. 12.

18. - Ibidem p. 213-214.

19. - Hannah Arendt, The human Condition, Chicago, 1958, trd. G. Fradier, 1961. Voir également la distinction que fait Eric Weil entre «Euvre » et «Action» dans Logique de la philosophie, Paris, 1950.

20. - Kant, Zum ewigen Frieden, 1795.

21. - La Bombe atomique et l'avenir de l'humanité, p. 293.

22. - Origine et sens de l'histoire, p. 267.

23. - Liberté et réunification, p. 73.

24. - Cf. Wolgang Rudzio, «Gefährdung der Freiheit - Karl Jaspers als politischer Schriftsteller», in Philosophie der Freiheit, Oldenburg, 1983.

25. - Autobiographie philosophique, p. 152-153.

26. - Sur le différend, à la fois personnel et philosophique, entre Heidegger et Jaspers, voir Jean Paumen, "Jaspers et Heidegger ", in Revue internationale de philosophie, 1983, et J. Lavaud, "Le différend Jaspers-Heidegger : du dialogue à l'anathème ", in Jaspers témoin de son temps, Bordeaux, 1986.

27. - Voir les hommages rendus à Jaspers comme penseur politique, particulièrement : Hannah Arendt, « Karl Jaspers, éloges », in Men in dark time, New York, 1968, trad. Vies politiques, Paris, 1974, et Raymond Aron, « Karl Jaspers et la politique » in Commentaire, Paris, 1985.

\section{RÉSUMÉS}

À partir d'une philosophie, comprise initialement comme éclairement de l'existence, par laquelle il s'est rendu célèbre, Jaspers fait une critique radicale de la civilisation moderne dans La Situation 
spirituelle de notre époque, en 1930. Il montre que la rationalisation et la massification à l'œuvre dans la société tendent à la négation de la subjectivité. Or, l'individu ne peut se contenter d'être rouage dans un mécanisme, producteur-consommateur dans une économie, aussi abondante soitelle! Par cette critique Jaspers s'inscrit dans le mouvement général de la «Kulturkritik» allemande. Mais Jaspers n'est pas seulement un moraliste. Il élabore après 1945 une philosophie positive de l'histoire, où il montre, dans un sens qui n'est pas hégélien, les percées décisives de la raison et les avatars de la liberté dans l'histoire. La civilisation actuelle, désormais mondiale, est en proie à plusieurs dilemmes tragiques : liberté politique ou totalitarisme, paix juridique ou guerre atomique, foi ou nihilisme, que seule une conversion à la raison peut surmonter. Jaspers intervient également, par des prises de position publiques, dans l'actualité politique de la République Fédérale Allemande. Il analyse dès 1945 le délicat problème de la responsabilité du peuple allemand dans les crimes nazis. Il discute le difficile problème de la réunification des deux Allemagnes. Il critique les tendances oligarchiques des partis politiques et les accuse de confisquer la démocratie. Jaspers est bien témoin et acteur de son temps, temps d'une histoire tragique et troublée, où il a toujours lutté pour la clarté rationnelle, la liberté existentielle et la liberté politique.

Durch eine zuerst als Existenzerhellung begriffene Philosophie, die ihn berühmt machte, übt Jaspers in seinem Werk Die geistige Situation der Zeit im Jahre 1930 eine radikale Kritik an der modernen Zivilisation. Er zeigt, dass die Rationalisierung und die Vermassung, die in der Gesellschaft tätig sind, auf die Negation der Subjektivität hingehen. Aber das Individuum kann sich nicht einfach damit begnügen, Triebwerk in einem Apparat, Erzeuger-Verbraucher in einer Wirtschaft zu sein. Diese Kritik Jaspers ist Bestandteil der allgemeinen Bewegung der deutschen Kultur kritik. Aber Jaspers ist nicht nur ein Moralist. Nach 1945 bekennt er sich zu einer wirklichen Philosophie der Geschichte, in der er, in einem nicht hegelschen Sinn, die entscheidenden Durchbrüche der Vernunft und die Abenteuer der Freiheit in der Geschichte zeigt. Die gegenwärtige, weltweite Zivilisation steht vor mehreren tragischen Entscheidungen: politische Freiheit oder Totalitarismus, völkerrechtlicher Friede oder Atomkrieg, Glaube oder Nihilismus, die nur durch eine Umkehr zur Vernunft überwunden werden können. Auch greift er, mit seinen öffentlichen Stellungnahmen, in die politische Aktualität der Bundesrepublik ein. Schon 1945 studiert er das heikle Problem der Verantwortung des deutschen Volkes in den NaziVerbrechen. Er erörtert das schwierige Problem der Wiedervereinigung der beiden Deutschland. Er bespricht die oligarchischen Tendenzen der Parteien und wirft ihnen vor, sich als einzige Vertreter der Demokratie zu betrachten. Jaspers ist ja der Zeuge und der Mitwirker seiner Zeit, einer tragischen und verzerrten Geschichte, in der er immer um die rationale Klarheit, die existentielle und die politische Freiheit gekämft hat.

\section{AUTEUR}

\section{JEAN-FRANÇOIS ROBINET}

Lycée Gaston Berger 